\title{
A high throughput instrument to measure mechanical losses in thin film coatings
}

G. Vajente, A. Ananyeva, G. Billingsley, E. Gustafson, A. Heptonstall, E. Sanchez, and C. Torrie

Citation: Review of Scientific Instruments 88, 073901 (2017); doi: 10.1063/1.4990036

View online: http://dx.doi.org/10.1063/1.4990036

View Table of Contents: http://aip.scitation.org/toc/rsi/88/7

Published by the American Institute of Physics

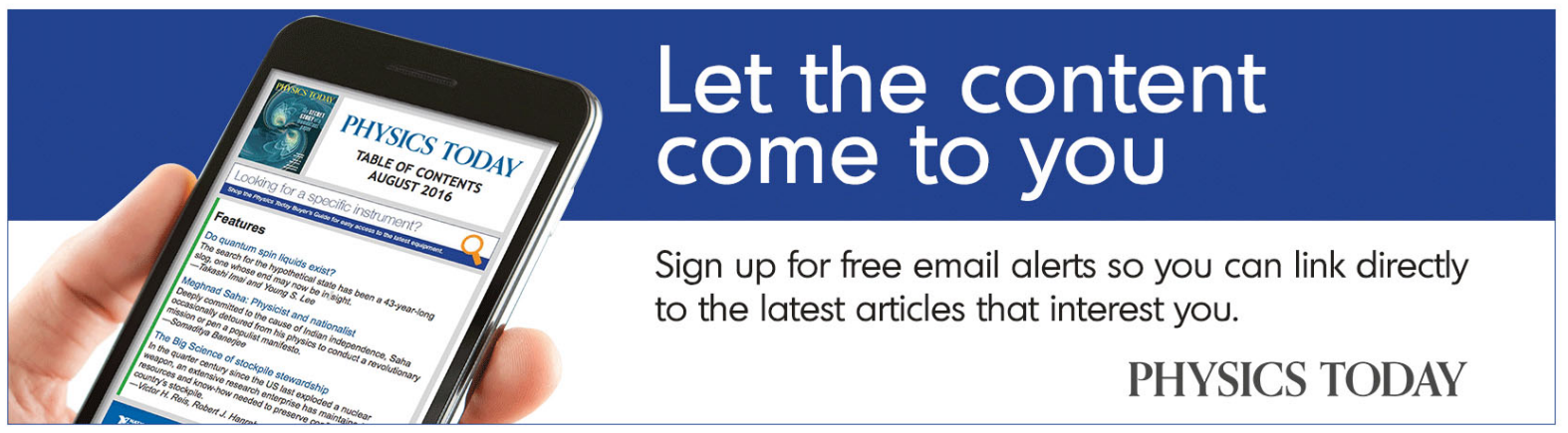




\title{
A high throughput instrument to measure mechanical losses in thin film coatings
}

\author{
G. Vajente, ${ }^{\text {a) }}$ A. Ananyeva, G. Billingsley, E. Gustafson, A. Heptonstall, E. Sanchez, \\ and C. Torrie \\ LIGO Laboratory, California Institute of Technology, Pasadena, California 91125, USA
}

(Received 25 April 2017; accepted 13 June 2017; published online 5 July 2017)

\begin{abstract}
Brownian thermal noise generated by mechanical losses in thin film coatings limits the sensitivity of gravitational wave detectors, as well as several high precision metrology experiments. Improving the sensitivity of the next generation of gravitational wave detectors will require optical coatings with significantly reduced mechanical losses. In this paper, we describe a system that we developed to measure the mechanical loss angle of thin film coatings deposited on fused silica substrates. The novelty of this system resides in the capability of parallel measurement of up to four samples and the ability to simultaneously probe all the resonant modes of each sample. This high throughput measurement system allows the exploration of a large number of deposition and material parameters, which can be tuned to achieve low loss coatings. Published by AIP Publishing. [http://dx.doi.org/10.1063/1.4990036]
\end{abstract}

\section{INTRODUCTION}

Advanced gravitational wave detectors, such as Advanced LIGO $^{1}$ and Advanced Virgo, ${ }^{2}$ will be limited, at their design sensitivity, by Brownian noise in the high reflectivity multilayer thin film coatings deposited on their test masses. ${ }^{3}$ To be able to improve the sensitivity of those instruments by about a factor of two, ${ }^{4}$ without large scale modifications of the present facilities, will require four times lower total mechanical losses in the coating stack. In the state-of-the-art high reflectivity coating used today in Advanced LIGO, two amorphous materials are used in alternated layers deposited by ion beam sputtering: silica $\left(\mathrm{SiO}_{2}\right)$ and titania-doped tantala ( TiO- $\left.\mathrm{Ta}_{2} \mathrm{O}_{5}\right)$. The measured mechanical loss angle of silica is $4 \times 10^{-5} \mathrm{rad}^{5}$ and that of doped tantala is $2.4 \times 10^{-4} \mathrm{rad}^{3}$ Therefore, the doped tantala layers dominate the mechanical losses, but to gain a global reduction of a factor 4 in the coating losses, silica also needs improvement.

The materials and doping levels used in the current coatings are the results of many years of research, exploring different deposition techniques and materials. ${ }^{3,6-10}$ There are many possible approaches to further improve the coating mechanical loss: use of other amorphous materials, ${ }^{11}$ including different doping concentrations and substances; different deposition techniques, such as elevated substrate temperature, ${ }^{12}$ dual ion beam deposition, ${ }^{13}$ and slow deposition rate; and use of crystalline coating materials. ${ }^{14}$ It is apparent that the space of all possible parameters to be explored is extremely large, and therefore there is a need for a system which is able to quickly and reliably measure a large number of coated samples. Indeed, a typical coating experiment can involve deposition of thin films on about 12 substrates. Each sample must be characterized before and after the deposition. Moreover, each sample might be subject to

\footnotetext{
a) vajente@ caltech.edu
}

multiple heat treatments after deposition, and the mechanical losses must be measured after each step. In conclusion, we envision the need of at least 50 measurements per coating experiment, with a cadence of about one experiment per month.

Our system is based on the gentle nodal suspension, initially developed at the Università di Firenze in Italy ${ }^{15}$ and then at the Laboratoire des Matériaux Avancés in Lyon, France. ${ }^{16}$ It is capable of measuring up to four coated samples at the same time, with a turnaround time of only few hours for each measurement. In Sec. II, we describe the principles of the suspension system and the details of our implementation, including the optical lever readout and the data acquisition system. Another key point of our experimental apparatus is that it is completely automated, from the measurement to the data reduction. In Sec. III, we describe the algorithms used to extract the mechanical loss angle from the raw experimental data.

\section{MEASUREMENT SYSTEM}

The mechanical loss of a coating can be obtained by depositing the thin film on a known substrate, then exciting the resonant modes of the system, and measuring the ring-down time. The standard substrates that we use for our ring-down measurements are disks (diameter $75 \mathrm{~mm}$, thickness $1 \mathrm{~mm}$ ) made of fused silica (Corning $7980^{17}$ or Suprasil $313^{18}$ ). The resonant modes of a substrate with this shape can be computed from analytical expressions ${ }^{19}$ based on a thin membrane approximation or using finite element modeling. We chose the latter approach, using COMSOL Multiphysics, ${ }^{20}$ since it provided us with more accurate predictions of the mode frequencies. Also, it can easily account for the presence of a thin layer of different materials on the surface, corresponding to the deposited coating. The first few mode frequencies and shapes are shown in Fig. 1. 

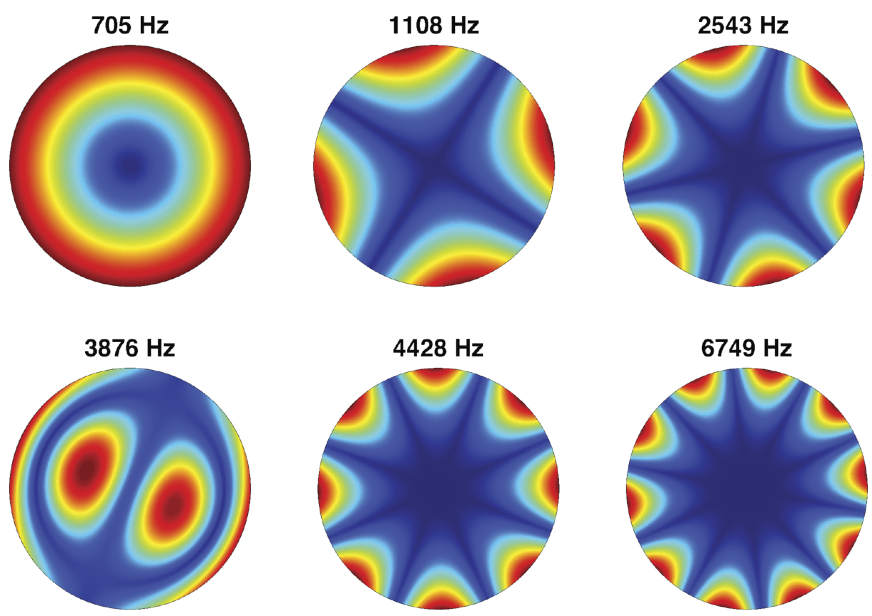

\section{A. Suspension}

The principle behind the gentle nodal suspension is to rest the substrate on the top of a spherical surface, in our case a silicon convex lens with a radius of curvature of about 60 $\mathrm{mm}$ (see Fig. 2). If the disk is centered, gravity and friction ensure that it will not slip. Indeed it can be shown ${ }^{15}$ that the horizontal position of the disk is a stable equilibrium position, provided that the radius of curvature of the spherical surface $R$ is larger than one half of the disk thickness $t: R>t / 2$. If the static friction between the disk and the spherical surface is large enough, the stable oscillation range can be very large

$$
\theta \lesssim \sqrt{3 \frac{2 R-t}{t}} .
$$

The contact region at the center of the disk is very small, and the resulting additional losses are negligible for all modes that do not have a significant deformation in the center (see Fig. 1). This constraint is not severe since it leaves tens of modes that can be excited and measured at frequencies between $1 \mathrm{kHz}$ and $30 \mathrm{kHz}$.

The disk modes are excited using a non-contacting electrostatic comb actuator: ${ }^{21}$ a series of parallel electrodes are held at about $1 \mathrm{~mm}$ from the disk surface; the electrodes are connected alternating to ground and to a high voltage amplifier,

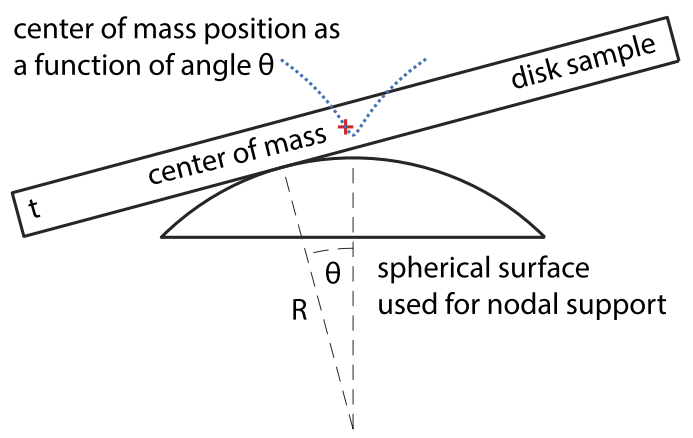

FIG. 2. Scheme of the gentle nodal suspension: the substrate is a disk with thickness $t$ resting on top of a fixed spherical surface of radius $R$. The orange cross shows the position of the disk center of mass for the inclination angle shown. The blue dots show the center of mass position as a function of the inclination angle, proving the stability of the configuration. Disk and sphere dimensions are not to scale with the real system. The disk inclination is exaggerated.
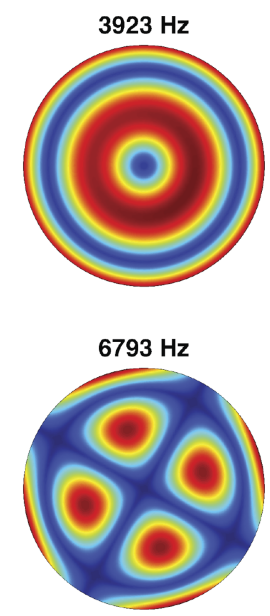

FIG. 1. Example shapes of the first few modes of the substrates used in the measurement system. The color shows the absolute value of the displacement in a direction orthogonal to the surface. The modes with a significant displacement or deformation at the center (such as those at 705, 3876, and $3923 \mathrm{~Hz}$ ) are not measurable in our system since they exhibit a large contact loss with the suspension.

able to source up to $4 \mathrm{kV}$ peak to peak with a bandwidth of several tens of $\mathrm{kHz}$. By driving the amplifier with broadband white noise, we can simultaneously excite all of the disk modes (about 20 with frequencies below $30 \mathrm{kHz}$ ).

Four identical realizations of the suspension system are mounted into a single vacuum chamber (see Fig. 3). We normally operate the chamber at a pressure below $10^{-6}$ Torr, to

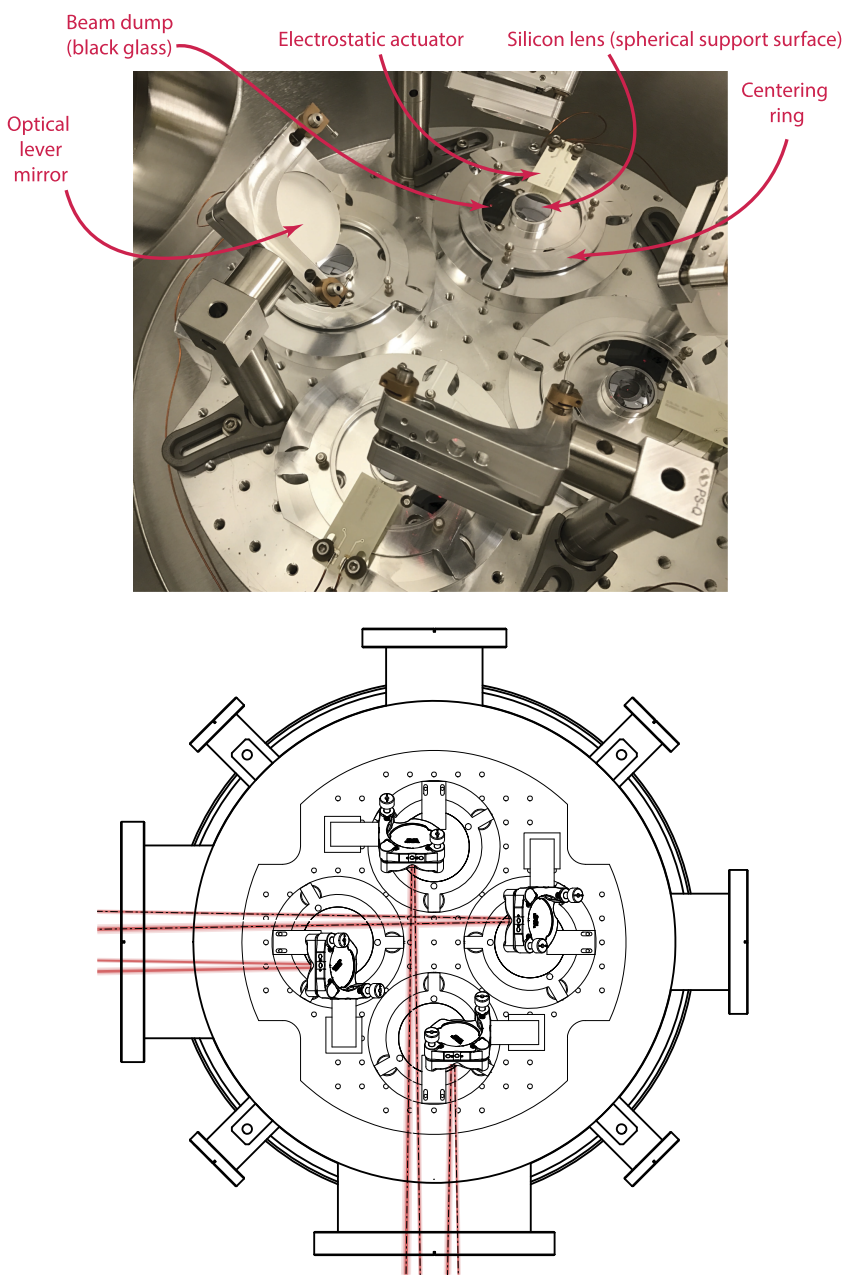

FIG. 3. Top: photo of the four measurement systems mounted inside the vacuum chamber. The main components are labeled. Bottom: optical layout of the four measurement systems, seen from the top. The laser beams are in red. 


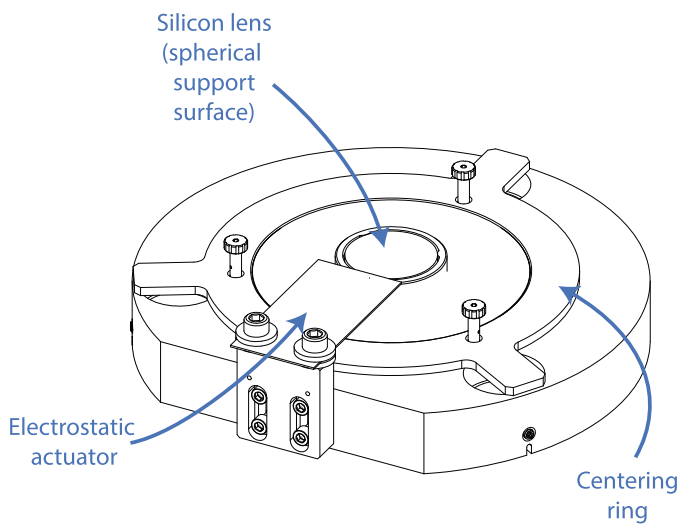

FIG. 4. Drawing of a single nodal suspension system, highlighting the main components of the system.

avoid damping due to residual air pressure. The entire system is inside a class 100 clean room.

To ease the installation and the centering of the disks on the nodal support, we added a centering ring to each of the four systems (see Fig. 4). This ring is precisely machined and can be moved up and down either manually or with a remote controlled translation stage. To install a disk, the centering ring is raised and the disk sits into an inset machined to match its dimensions. The centering ring is then gently lowered, making contact between the disk and the nodal support. In normal operating conditions, the ring rests at its lower position, avoiding any interference with the disk being measured.

\section{B. Optics}

Each disk surface motion is measured using an optical lever. A picture of the actual setup is shown in Fig. 5. Four $\mathrm{HeNe}$ laser beams are sent into the vacuum chamber and they are steered by four mirrors to hit the upper surface of the disks. The precise position is not crucial, but it is important to be

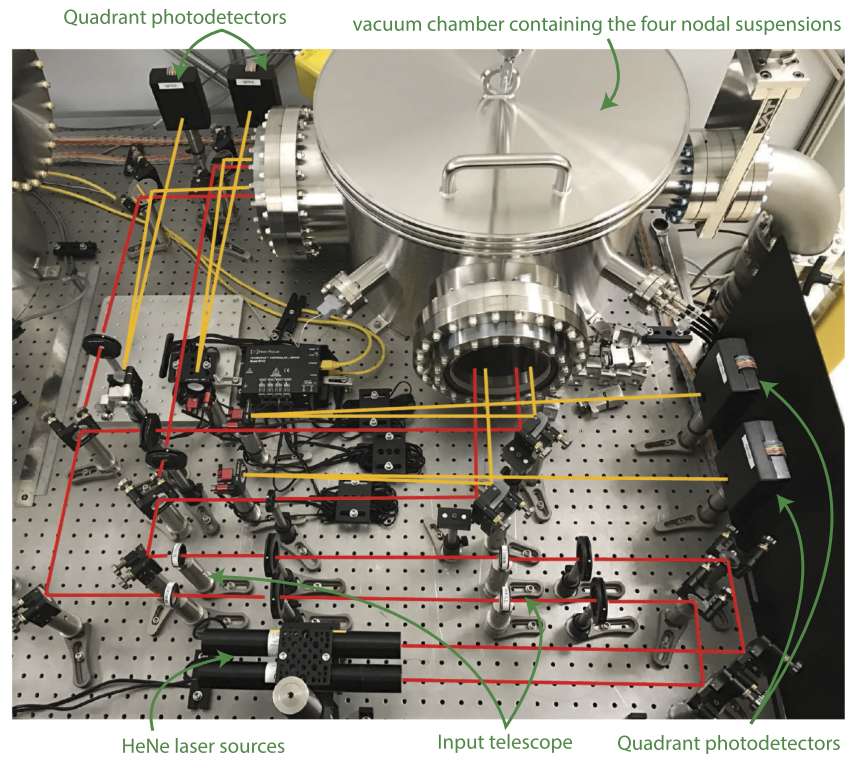

FIG. 5. Photo of the optical lever setup. The beams going into the vacuum chamber, to the four measurement systems, are highlighted in red. The beams coming out of the chamber and incident on the quadrant photodetectors are highlighted in yellow. close to the edge, where the resonant modes induce a larger displacement. The reflected beams are extracted from the vacuum chamber and impinge on silicon quadrant split photodetectors (QPDs, Hamamatsu S598122). The power readouts from the four sectors of each QPD are combined to give X and Y beam spot motion signals, which are in turn divided by the total power on the QPD, to obtain a dimensionless normalized beam spot position signal which is proportional to the angular motion of the disk surface. A two lens telescope is used on the input beam to ensure that the beam radius on the QPD is about $300 \mu \mathrm{m}$. This allows us to reach a conversion factor of $1.6 \times 10^{4} \mathrm{rad}^{-1}$ for the disk angular motion into the normalized dimensionless QPD signals. We typically measure $100 \mu \mathrm{W}$ of power impinging on each QPD, resulting in a shot noise limited sensitivity to disk surface motion of the order of $10^{-11}$ $\mathrm{rad} / \sqrt{\mathrm{Hz}}$ above $2 \mathrm{kHz}$. Below this frequency, the QPD signals are dominated by seismic and acoustic noises due mainly to the clean room air filters.

The beam which is transmitted through the disk is partially dumped by a slab of black glass, slightly tilted, in such a way that the residual reflection is extracted from the vacuum chamber and properly dumped on the optical table.

The beams impinging on the QPDs are steered using remotely controlled picomotor mounts. This ensures precise centering of the beams on the sensors during each measurement.

\section{Data acquisition and automation}

The signals generated by the four sectors of each QPD are digitized by 16 bit analog-to-digital converter modules (General Standards model 16AI32SSC ${ }^{23}$ ) and are available for real time processing and storage. The digital data acquisition and processing system is a custom stand-alone version of the standard Advanced LIGO Control and Data System,${ }^{24}$ running at a sampling frequency of $65 \mathrm{kHz}$. This high sampling frequency allows us to simultaneously measure the disk motion induced by all modes below $32.5 \mathrm{kHz}$. The combination of the QPD quadrant signals is done digitally in the real time system. We also have access to analog output channels (using a General Standards digital-to-analog converter model 16AO16 ${ }^{23}$ ). Those channels are used to drive the electrostatic actuators. We have the capability of producing arbitrary waveforms, but we use wide-band random noise to excite all the disk modes simultaneously.

The normalized QPD signals, as well as the total power impinging on the QPDs, are archived to a disk and are used for the offline data reduction described in Sec. III.

All parameters of the digital control system are accessible using scripting languages, such as python. ${ }^{25}$ This allows us to automate the measurement procedure.

\section{Cross talk between samples}

Having four samples measured simultaneously in the same vacuum chamber raises naturally the question of cross talks between the four suspensions. There are two main sources of possible cross talks: vibrations of one the samples might propagate through the gentle nodal suspension to another sample or the signals read by different QPDs might mix at the 


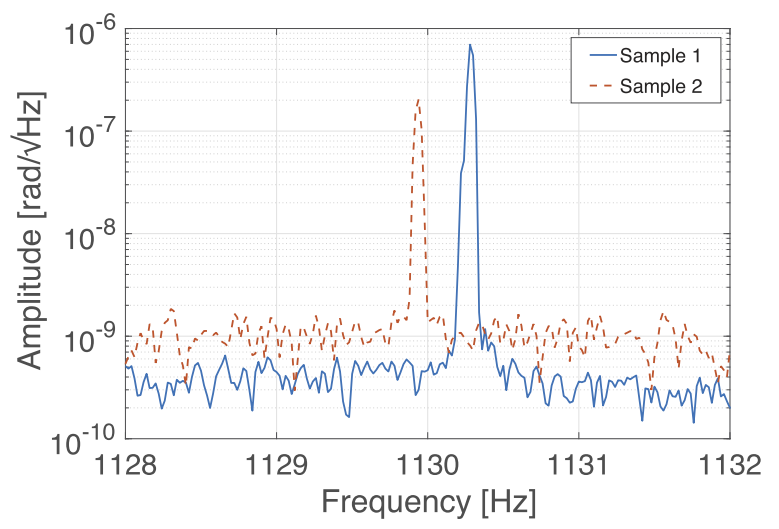

FIG. 6. QPD signals, zoomed around one of the resonant modes, for two samples that have been excited simultaneously and are being measured at the same time. Each QPD signal is clearly sensitive to vibration of only one of the samples, proving that there is no mechanical or electronic cross talk, at a level of at least $1 / 1000$.

electronics level. Both sources of cross talk were experimentally excluded with very good accuracy. Figure 6 shows the spectra of the QPD signals from two different samples, right after the modes of both samples have been excited: although the separation between the modes is relatively small, there is no sign of any cross talk, at a level of at least 1/1000.

\section{DATA REDUCTION}

A typical measurement proceeds as follows. The automation script disables all QPD centering servos (to avoid inducing spurious vibrations) and logs the time of a quiet period of data. Then the excitation is switched on for a variable period of time, from 10 to $60 \mathrm{~s}$. The starting and ending times of the excitation are recorded. Then the script waits for a suitably long period of time (from tens of minutes to hours) for the disk modes to ring down, and if requested repeats the measurement several times. This whole procedure is implemented in a python script. The $\mathrm{X}$ and $\mathrm{Y}$ signals of each of the four QPDs are stored on disk, so that the data analysis can be performed offline, using a set of MATLAB ${ }^{26}$ programs.

Figure 7 compares the $\mathrm{X}$ and $\mathrm{Y}$ signals of one of the QPDs, before and after the excitation. The resonant modes of the disk are clearly visible in the post excitation spectrum. The first step in the data analysis is the automatic identification of all excited peaks. The ratio of the spectrum after the excitation divided by the spectrum before the excitation is used for this purpose: all frequency bins where the ratio is larger than a threshold (typically 10) are identified and clustered together, resulting in a list of all peaks that appeared after the excitation. The lowest frequency peak is at about $1.1 \mathrm{kHz}$. The precise frequency of this peak is measured and compared with a set of precomputed finite element analysis (FEA) simulations, to find the best match. The main parameter that is tuned in those simulations is the disk thickness. Other parameters have an effect which is either indistinguishable from the thickness ${ }^{19}$ (Young's modulus, diameter, and density) or very small (Poisson's ratio). The simulation that best matches the frequency of the first mode is then used to predict the frequency of all higher order modes and to select only the detected
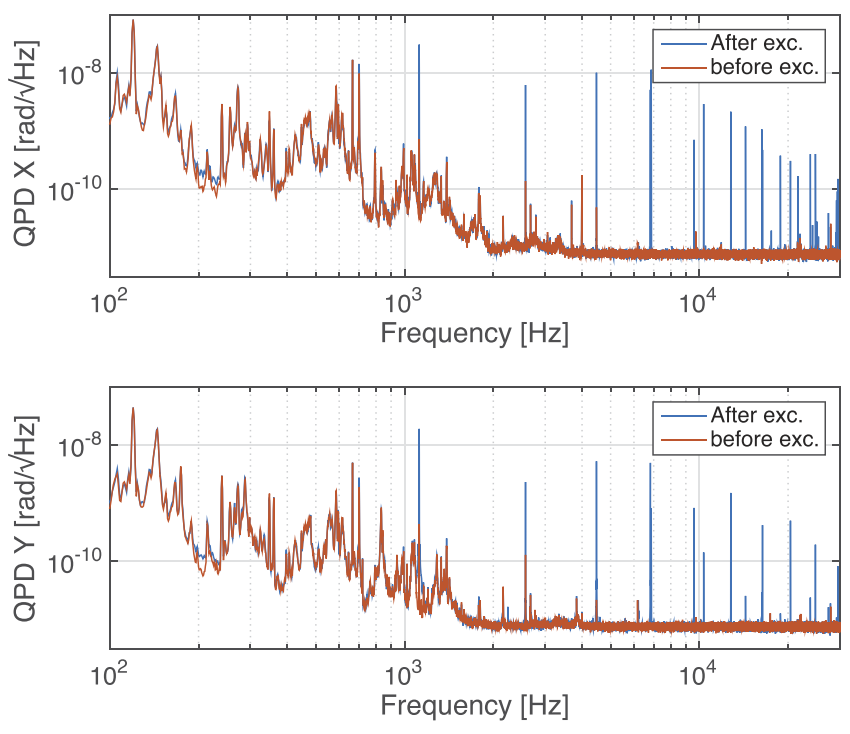

FIG. 7. Comparison of QPD signals before and after the excitation is applied. The spectrum after the excitation clearly shows all the resonant modes of the disk. The QPD signals are shot noise limited above $2 \mathrm{kHz}$, while below that frequency they are limited mainly by acoustic and seismic disturbances. However, it is clear from the amplitude of the peaks after the excitation that this noise background level is not a limitation for our purposes.

peaks that match those frequencies within a few Hz. This model based algorithm allows us to exclude spurious peaks that appear due to beating between the real modes and environmental disturbances, and to properly label each detected frequency with the mode family (number of radial and azimuthal nodes).

The amplitude of each of the identified peaks is then tracked by computing short ( $1 \mathrm{~s})$ Fourier transforms over a time period that can range from tens of minutes (if the expected sample losses are large) to hours (if the losses are low, as for uncoated substrates). In the case of a simple resonant mode, the time evolution of the amplitude follows an exponential decay

$$
A_{i}(t)=A_{i}(0) e^{-t / \tau_{i}},
$$

where the time constant $\tau_{i}$ is related to the mechanical losses $\phi_{i}$ and the quality factor $Q_{i}$ of the $i$ th mode by

$$
\tau_{i}=\frac{1}{\pi f_{i} \phi_{i}}, \quad Q_{i}=\frac{1}{\phi_{i}}=\pi f_{i} \tau_{i},
$$

$f_{i}$ being the mode frequency. The situation is a bit more complex in the case of our disk substrates: due to the rotational symmetry, each mode is actually an almost degenerate doublet. The frequency splitting of the two modes in each doublet, due to disk imperfections, can be very small: we measured typical values between 10 and $300 \mathrm{mHz}$. Therefore our analysis procedure, which has a bandwidth of $1 \mathrm{~Hz}$, cannot separate the contribution of the two modes. The decay is not a simple oscillation with an exponential envelope but rather the result of the beating of the two decaying modes, with random initial phase and amplitude, separated by a small frequency splitting. For each doublet, the $\mathrm{X}$ and $\mathrm{Y}$ signals detected by a QPD have the following form: 


$$
\begin{aligned}
q_{i}^{2}(t)= & \left|A_{i, 1} e^{2 \pi i f_{i} t-t / \tau_{i, 1}}+A_{i, 2} e^{2 \pi i\left(f_{i}+\Delta f_{i}\right) t-t / \tau_{i, 2}+\varphi_{i}}\right|^{2} \\
= & A_{i, 1}^{2} e^{-2 t \gamma_{i, 1}}\left[1+\frac{A_{i, 2}^{2}}{A_{i, 1}^{2}} e^{-2 t \Delta \gamma_{i}}+2 \frac{A_{i, 2}}{A_{i, 1}} e^{-t \Delta \gamma_{i}}\right. \\
& \left.\times \cos \left(2 \pi t \Delta f_{i}+\varphi_{i}\right)\right],
\end{aligned}
$$

where $\gamma_{i, 1}=1 / \tau_{i, 1}$ and $\gamma_{i, 2}=1 / \tau_{i, 2}$ are the inverse of the time constant of the two modes, $f_{i, 1}$ and $f_{i, 2}$ are their frequencies, $A_{i, 1}$ and $A_{i, 2}$ are the initial amplitudes of the two modes, and $\varphi_{i}$ is the initial relative phase. We denoted the frequency splitting with $\Delta f_{i}=f_{i, 1}-f_{i, 2}$ and the difference of inverse time constants with $\Delta \gamma_{i}=\gamma_{i, 1}-\gamma_{i, 2}$.

A typical example of the time evolution of the beating of the two modes in a doublet is shown in Fig. 8: as expected from Eq. (4), it consists of an oscillation at the splitting frequency with an exponentially decaying envelope.

To extract the mechanical quality factor of the two modes, we need to fit the experimental data with the full model described in Eq. (4), with six free parameters
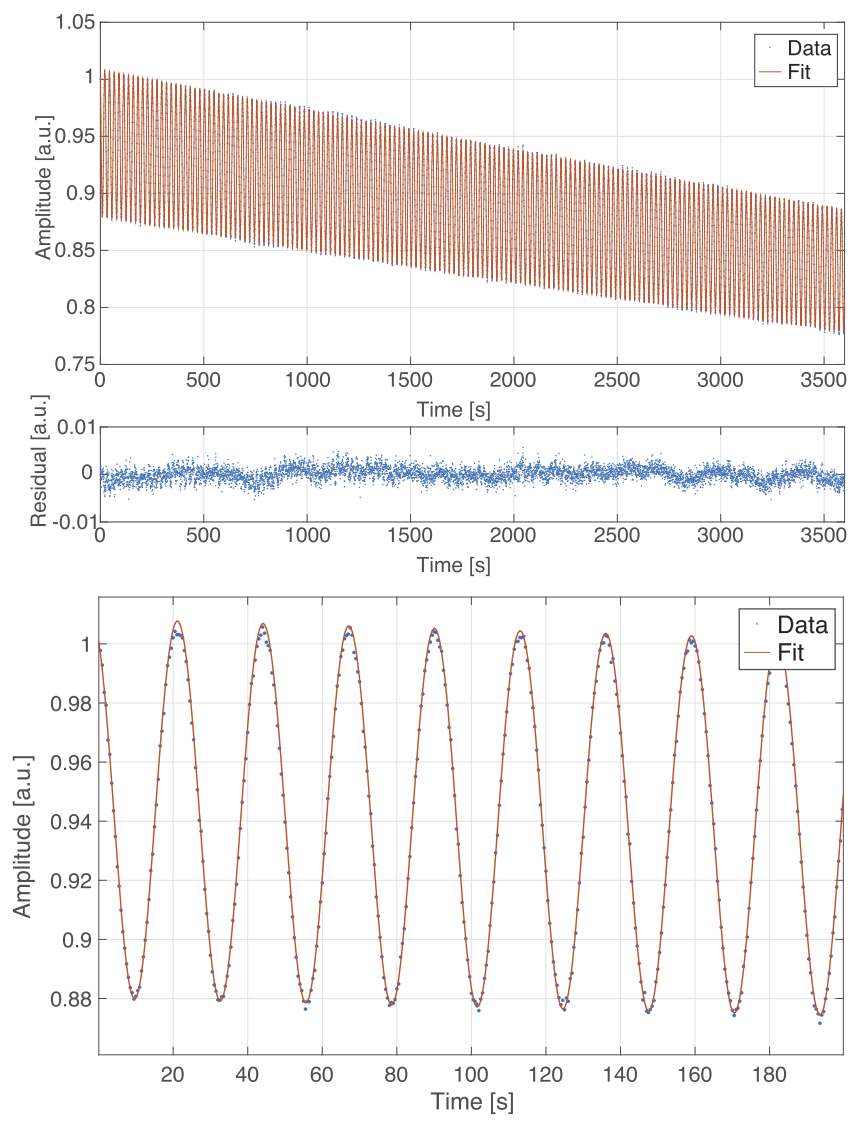

FIG. 8. Example of the time evolution of the detected mode amplitude in one of the QPD signals. In the top panel, dots (barely visible behind the solid line) represent the experimental data, and the solid line is the fit based on Eq. (4). The middle panel shows the fit residuals. In this case, the tracked peak is the first measurable mode (the second one in the first row of Fig. 1). The measured frequency is $f_{1}=1120.8 \mathrm{~Hz}$, the splitting is $\Delta f_{1}=44 \mathrm{mHz}$, and the two mechanical quality factors are equal within the measurement errors $Q_{2,1}=Q_{2,2}=1.00 \pm 0.02 \times 10^{8}$. In this case, the quality factor is so large that even over a period of $1 \mathrm{~h}$, the decay looks almost linear. The bottom panel shows a zoom into the first $200 \mathrm{~s}$ of the ring-down, to better show the oscillation due to the beat of the two almost degenerate modes and the good agreement between the data and fit.

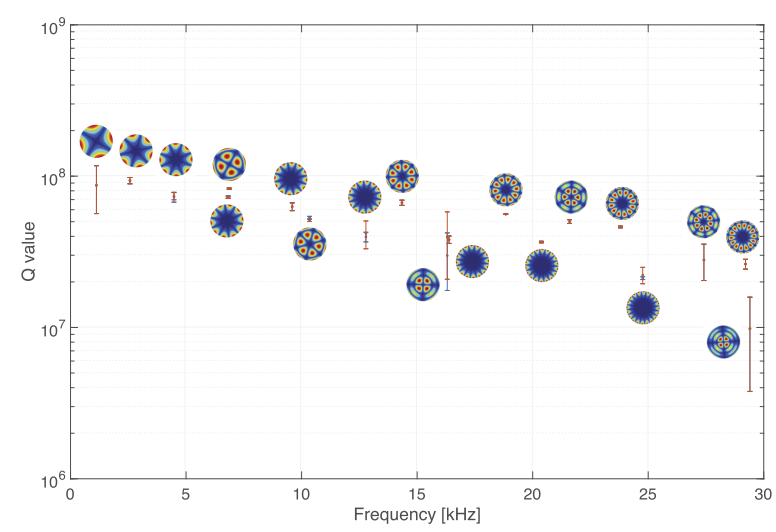

FIG. 9. An example of the final result of the measurement procedure and data analysis code showing the mechanical quality factor for all identified modes. For each frequency, there are two points, corresponding to the two modes in the degenerate pair. In most cases, the two points are undistinguishable. The error bars are the $95 \%$ confidence intervals computed from the fit. The images show the mode shape corresponding to each data point, as identified by the automatic script, based on finite element analysis.

$A_{i, 1}, A_{i, 1} / A_{i, 2}, \Delta f_{i}, \gamma_{i, 1}, \Delta \gamma_{i}, \varphi_{i}$. The fit is obtained by minimization of a least square cost function. The highly non-linear dependency of the cost function on the parameters makes this a difficult optimization problem, very sensitive to the initial guess. For this reason, the first step of our fitting algorithm is to estimate the initial point for the minimization. First, we fit the ring-down with a simple exponential, providing a good estimate of the two time constants $\gamma_{i, 1}$ and $\gamma_{i, 2}$. We initially assume that $\Delta \gamma_{i}=0$. Then we subtract the mean exponential envelope from the data and use the peak to peak value of the residual, together with the initial value of the exponential envelope, to estimate $A_{i, 1}$ and $A_{i, 2}$. A Fourier transform of the residual is also used to estimate the splitting frequency $\Delta f_{i}$ and the phase $\varphi_{i}$. With the initial parameters estimated in this manner, a non-linear optimization code is always able to converge to a good fit, as shown in Fig. 8. Confidence intervals are computed for all the parameters, based on the Jacobian matrix of the cost function near the optimal parameters. $^{27}$

The procedure described above is automatic and implemented in MATLAB. ${ }^{26}$ Figure 9 shows a typical result of the ring-down measurement of one of the uncoated substrates. In this case, 19 modes between $1 \mathrm{kHz}$ and $30 \mathrm{kHz}$ were identified and measured.

\section{CONCLUSION}

We described an instrument to measure the mechanical losses in coated or uncoated fused silica disk samples. The novel approaches we used in this system are motivated by the need to be able to measure a large number of samples in a short time, in order to explore a large volume in the space of deposition and material parameters, which might determine the coating mechanical losses. The final goal is to develop a low mechanical loss, high reflection dielectric coating for future upgrades of the Advanced LIGO detector. ${ }^{4}$

To achieve our goal, we installed four independent measurement systems inside the same vacuum chamber, thus significantly reducing the down time due to the pumping and 
venting procedure. Moreover, the use of the gentle nodal suspension, together with a movable centering ring, makes the installation and balancing of the samples easy and fast. Probably the most significant improvements with respect to other systems ${ }^{15,28,29}$ are our capability to excite and measure all the sample resonant modes at the same time ${ }^{30}$ and the completely automated mode identification and tracking.

All the improvements described in this paper allowed us to increase the throughput of mechanical loss measurement by almost two orders of magnitude with respect to what was previously achievable. We are typically able to measure four uncoated samples, with quality factors greater than $10^{7}$ in about $4 \mathrm{~h}: 2 \mathrm{~h}$ accounts for the sample installation and the vacuum chamber evacuation; $1-2 \mathrm{~h}$ is needed to measure a high quality factor ring-down; finally $0.5 \mathrm{~h}$ is needed to vent the vacuum chamber. We typically repeat the measurements at least 4 times for the same set of samples, meaning that we can complete a set of 4 consecutive measurements on 4 samples over a period of $8 \mathrm{~h}$. The only step that requires human intervention is the installation of the samples; therefore, we can completely characterize up to eight different coatings per day.

\section{ACKNOWLEDGMENTS}

The authors are grateful to M. Granata, G. Cagnoli, Q. Cassar, and J. Degallaix (Laboratoire des Matériaux Avancés) for the opportunity to work with a gentle nodal suspension system, and to S. Penn (Hobart and William Smith Colleges) for useful comments and suggestions. LIGO was constructed by the California Institute of Technology and Massachusetts Institute of Technology with funding from the National Science Foundation, and operates under cooperative Agreement No. PHY-0757058. Advanced LIGO was built under Award No. PHY-0823459. This paper carries LIGO Document No. LIGO-P1700078.

${ }^{1}$ J. Aasi et al., "Advanced LIGO,” Classical Quantum Gravity 32(7), 074001 (2015).

${ }^{2}$ F. Acernese et al., "Advanced Virgo: A second-generation interferometric gravitational wave detector," Classical Quantum Gravity 32(2), 024001 (2014).

${ }^{3}$ G. M. Harry et al., "Titania-doped tantala/silica coatings for gravitationalwave detection,” Classical Quantum Gravity 24, 405 (2006).
${ }^{4}$ J. Miller et al., "Prospects for doubling the range of advanced LIGO," Phys. Rev. D 91, 062005 (2015).

${ }^{5}$ S. D. Penn et al., "Mechanical loss in tantala/silica dielectric mirror coatings," Classical Quantum Gravity 20, 2917 (2003).

${ }^{6}$ R. Flaminio et al., "A study of coating mechanical and optical losses in view of reducing mirror thermal noise in gravitational wave detectors," Classical Quantum Gravity 27, 084030 (2010).

${ }^{7}$ J. Steinlechner et al., "Thermal noise reduction and absorption optimization via multimaterial coatings," Phys. Rev. D 91, 042001 (2015).

${ }^{8} \mathrm{~J}$. Steinlechner et al., "Optical absorption of ion-beam sputtered amorphous silicon coating," Phys. Rev. D 93, 062005 (2016).

${ }^{9}$ M. Granata et al., "Mechanical loss in state-of-the-art amorphous optical coatings," Phys. Rev. D 93, 012007 (2016).

${ }^{10} \mathrm{G}$. D. Cole et al., "Tenfold reduction of Brownian noise in high-reflectivity optical coatings," Nat. Photonics 7, 644 (2013).

${ }^{11} \mathrm{~S}$. Reid and W. Martin, "Development of mirror coatings for gravitational wave detectors," Coatings 6, 61 (2016).

${ }^{12}$ X. Liu et al., "Hydrogen-free amorphous silicon with no tunneling states," Phys. Rev. Lett. 113, 025503 (2014).

${ }^{13}$ J. J. Cuomo, H. R. Rossnagel, and H. R. Kaufman, Handbook of Ion Beam Processing Technology (Noyes Publications, 1989).

${ }^{14}$ G. D. Cole et al., "High-performance near- and mid-infrared crystalline coatings," Optica 3(6), 647 (2016).

${ }^{15}$ E. Cesarini et al., "A 'gentle' nodal suspension for measurements of the acoustic attenuation in materials," Rev. Sci. Instrum. 80, 053904 (2009).

${ }^{16} \mathrm{M}$. Granata et al., "Internal friction and Young's modulus measurements on $\mathrm{SiO}_{2}$ and $\mathrm{Ta}_{2} \mathrm{O}_{5}$ films done with an ultra-high $\mathrm{Q}$ silicon-wafer substrate," Arch. Metall. Mater. 60, 365 (2015).

${ }^{17}$ See https://www.corning.com for Corning HPFS Fused silica 7980.

${ }^{18}$ See https://www.heraeus.com/ for Heraeus Fused Silica Suprasil 313.

${ }^{19} \mathrm{M}$. Amabili et al., "Natural frequencies and modes of free-edge circular plates vibrating in vacuum or in contact with liquid," J. Sound Vib. 188 , 685 (1995).

${ }^{20}$ See https://www.comsol.com for COMSOL Multiphysics.

${ }^{21}$ A. Cadez et al., "Measuring high mechanical quality factors of bodies made of bare insulating materials," J. Phys. E: Sci. Instrum. 21, 453 (1988).

${ }^{22}$ See http://www.hamamatsu.com for Hamamatsu Si PIN photodiode model S5981.

${ }^{23}$ See http://www.generalstandards.com for General Standards Corporation.

${ }^{24} \mathrm{R}$. Bork and A. Ivanov, "New control and data acquisition system in the advanced LIGO project," LIGO document No. P1100052, 2011.

${ }^{25} \mathrm{See}$ https://www.python.org for Python Software Foundation.

${ }^{26}$ See https://www.mathworks.com for Mathworks MATLAB.

${ }^{27}$ G. A. F. Seber and C. J. Wild, Nonlinear Regression (Wiley-Interscience, 2003).

${ }^{28}$ D. R. M. Crooks et al., "Experimental measurements of mechanical dissipation associated with dielectric coatings formed using $\mathrm{SiO}_{2}, \mathrm{Ta}_{2} \mathrm{O}_{5}$ and $\mathrm{Al}_{2} \mathrm{O}_{3}$," Classical Quantum Gravity 23, 4953 (2006).

${ }^{29} \mathrm{~A}$. V. Cumming et al., "Measurement of the mechanical loss of prototype $\mathrm{GaP} / \mathrm{AlGaP}$ crystalline coatings for future gravitational wave detectors," Classical Quantum Gravity 32, 035002 (2015).

${ }^{30}$ The system currently used at the Laboratoire des Matériaux Avancés is also capable of measuring multiple modes simultaneously. 